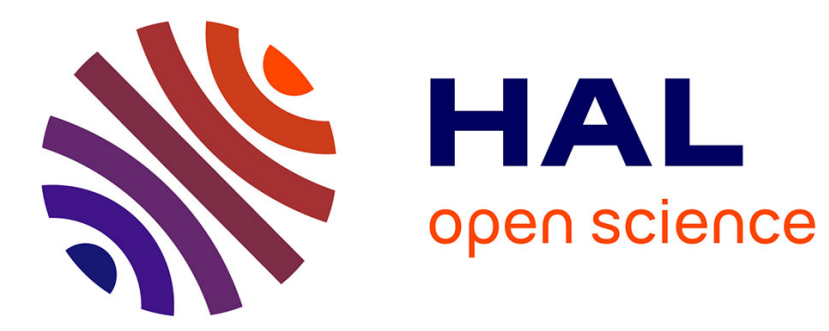

\title{
M-functions and parallel asynchronous algorithms
}

Didier El Baz

\section{To cite this version:}

Didier El Baz. M-functions and parallel asynchronous algorithms . SIAM Journal on Numerical Analysis, 1990, 27 (1), pp. 136-140. hal-01152707

\section{HAL Id: hal-01152707 https://hal.science/hal-01152707}

Submitted on 18 May 2015

HAL is a multi-disciplinary open access archive for the deposit and dissemination of scientific research documents, whether they are published or not. The documents may come from teaching and research institutions in France or abroad, or from public or private research centers.
L'archive ouverte pluridisciplinaire HAL, est destinée au dépôt et à la diffusion de documents scientifiques de niveau recherche, publiés ou non, émanant des établissements d'enseignement et de recherche français ou étrangers, des laboratoires publics ou privés. 


\title{
$M$-FUNCTIONS AND PARALLEL ASYNCHRONOUS ALGORITHMS*
}

\author{
DIDIER EL BAZ†
}

\begin{abstract}
The solution of nonlinear systems of equations $f x=z$ via parallel asynchronotis algorithms is considered. It is shown that when $F$ is continuous, oli-diagonaly ant itone, and strictly diagorally isolone, then point asynchronous sterations converge monolonically to a solution of the probem from supersolutions and subsolutions. A globial convergence result for asynchronous jterations, when $F$ is a continuous, surjective $M \cdot$ function is also presented.
\end{abstract}

Key Hords. nonlinear systems of equations, $M$-functions, paraltel computation, asynchronous iterations

AMS(MOS) sabject slassifications. 65 W05, 65H10, 65 N20

1. Introduction. There is a variety of parallel iterative methods for nonlinear problems (see Baudet [1], Schendel [12, App. 2], Sloboda [13]). In this paper we consider asynchronous relaxation methods for nonlinear systems of equations. There is now considerable understanding of the convergence propertics of parallel asynchronous iterations for a broad class of problems including some linear and nonlinear systems of equations, network flow problems, and dynamic programming. First, Chazan and Miranker [4] have formulated a model of parallel asynchronous algorithms. They have shown that parallel asynchronous iterations converge to the solution of a linear system of equations $A x=b$ if and only if $A$ is an $H$-matrix. Donnelly [5] has given convergence results for overrelaxed periodic schemes in the linear case. Mieltou [6] and Baudet [1] have extended the results of Chazan and Miranker to nonlinear fixed-point problerns by proving the convergence of parallel asynchronous algorithms for $P$-contraction mappings. Concurrently, Miellou [7] has shown that asynchronous iterations converge monotonically from supersoivtions and subsolutions for continuous, isotone fixed-point mappings. Bertsekas [2] has also shown the monotone convergence of a distributed asynchronous algorithm for a broad class of dynamic programming problems. In a recent paper [3], Bertsekas and El Baz obtained the same result for single commodity convex network flow probtems. In these last two papers the convergence is based on the property of isotonicily of the fixed-point mappings. Finally, Miellou [8] has considered the nonlinear system of equations $F x=z$ and some corresponding fixed-point mapping $G$ for block asynchronous iterations, and has shown that when $F$ is a continuous, surjective $M$-function, then $G$ is isotone. Moreover, if $G$ is continuous, then asynchronous iterations converge monotonically from supersolutions and subsolutions.

In this paper we concentrate on point asynchronous iterations. We show that when $F$ is continuous, off-diagonally antitone, and strictly diagonally isotone, then asynchronous iterations converge monotonically from supersolutions and subsolutions. We show also that any asynchronous iteration converges to the unique solution of $F X=z$, whatever the value of $z$, and for any starting point when $F$ is a continuous surjective $M$-function. The results presented in this paper extend to asynchronous iteration convergence results for underrelaxed Gauss-Seidel and Jacobi iterations proved by Rheinboldt $[10, \S 3]$. For other extensions of the convergence results in [10], in particular to block processes, the reader is referred to Rheinboldt $[11, \$ 6]$. 
The class of problems considered in this study is broad. Oft-diagonally antitone mappings and $M$-functions occur in the discretization of certain boundary value problems and in the study of nonlinear network flows (see [10], [1] ]).

In $\$ 2$ we introduce a fixed-point problem associaled with the nonlinear system of equations $F_{X}=z$ and study the propertics of the fixed-point mapping when $F$ is continuous, off-diagonally antitonc, and strictly diagonally isotone. In 3 we present convergence results for asynchronous iterations.

2. Preliminaries. We consider the solution of nonlinear systems of equations

$$
F x=\left(\begin{array}{c}
f_{1}\left(x_{1}, \cdots, x_{n}\right) \\
\cdots \\
f_{n}\left(x_{1}, \cdots, x_{n}\right)
\end{array}\right)-\left(\begin{array}{c}
z_{1} \\
\cdots \\
z_{n}
\end{array}\right),
$$

where $x_{1}, \cdots, x_{n}$ denote the components of vector $x$ element of the $n$-dimensional real linear space $R^{n}$. The natural partial ordering on $R^{\prime \prime}$ is defined by

For $x, y \in R^{n}, \quad x \leqslant y \quad$ if and only if $x_{i} \leqq y_{i}, \quad i=1, \cdots, n$.

LEMMA 2.1. Let $F: D \subset R^{n} \rightarrow R^{n}$ be continuous, off-diagonally anitione, and siricily diagonally isotone, and suppose that for some $z \in R^{\prime \prime}$ there exist points $x^{0}, y^{0} \in D$ such that

$$
x^{0} \leqq y^{\prime \prime}, \quad D^{\prime}=\left\{x \in R^{n} \mid x^{t} \leq x \leq y^{t h}\right\} \subset D, \quad F x^{0} \leqq z \leq F y^{0}
$$

Then, for any $x \in D^{\prime}$ there exists a linique vector $\hat{x} \in D^{\prime}$ with components $\hat{x}_{1}$ for which

$$
f\left(x_{1}, \cdots, \hat{x}_{t}, \cdots, x_{n}\right)=z_{1}, \quad i=1, \cdots, n \text {. }
$$

Proof. Suppose that for $x \in D^{\prime}$ and $i \in\{1, \cdots, n\}, f(x)<z_{i}$. Since $F$ is offdiagonaly antitore and $x \leqslant y^{\circ}$, it follows that (see [10, Def. 2.7])

$$
f_{f}(x)<z_{1} \text { 菃 } f_{i}\left(y^{(1)}\right) \cong f_{1}\left(x_{1}, \cdots, y_{1}^{0}, \cdots, x_{n}\right) \text {. }
$$

By the continuity and strict diagonal isotonicity of $F$ (see [10, Def. 2.7]), (2.2) implies the existence of a unique $\hat{x}_{1}$ for which

$$
x_{t} \leqq \hat{x}_{1} \leqq y_{1}^{0} \text { and } f\left(x_{1}, \cdots, \hat{x}_{i}, \cdots, x_{n}\right)=z_{1} .
$$

For further details about off-diagonaliy antitone and strictly diagonally isotone mappings the teader is referred to Ortega and Rheinboldt $[9, \$ 13.5]$ and Rheinboldt $[10$, $\$ 2],[11, \$ 2]$.

Now suppose that $f_{i}(x) \geq z_{r}$. From the oft-diagonal antitonicity of $F$ it lollows that

$$
f_{i}\left(x_{1}, \cdots, x_{1}^{0}, \cdots, x_{n}\right) \leqq f_{i}\left(x^{0}\right) \leq z_{1} \leqq f_{i}(x) \text {. }
$$

By the contimuity and strict diagonal isotonicity of $F,(2.3)$ implies the existence of a unique $\hat{x}_{1}$ lor which

$$
x_{1}^{\prime \prime} \subseteq \hat{x}_{1} \leq y_{i}^{\prime \prime} \text { and } f\left(x_{1}, \cdots, \hat{x}_{i}, \cdots, x_{n}\right)=z_{i}
$$

We introduce the fixed-point mapping $G: D^{\prime} \subset R^{\prime \prime} \rightarrow D^{\prime}$ defined by

$$
G x=\hat{x} \text {, where } \hat{x} \text { is defined by (2.1). }
$$

Clearly, $G$ is well defined; moreover, $x^{*}$ is a fixed point of $G$ if and only if $F x^{*}=z$.

LEMMA 2.2. Under the hypothesis of Lemma 2.1, the fixed-point mapping $G$, which is defined by (2.4), is continuous and isotone on $D^{\prime}$. Moreover, $x^{0} \leq G x^{n}, G y^{0} \leqslant y^{\circ}$ for starting points $x^{0}$ and $y^{\circ}$ for which $F x^{0} \leq z \leq F y^{\circ}$.

This result is derived from the proof of Theorem 6.3 of [11]. $x^{11}$ and $y^{\prime \prime}$ are a so-called subsolution and supersolution, respectively. 
In the notational conventions of this paper a subscript denotes a component index and a superscript denotes an iteration index.

3. Convergence of asynchronous iterations. We consider asynchronous iterations for the solution of systems of $n$ equations $F x=z$.

In brief, an asynchronous iteration relative to the solution of $F_{x}=z$, the starting point $x^{0}$, the sequence of delays $\left\{k^{s}=\left(k_{1}^{p}, \cdots, k_{n}^{p}\right)\right\}$, and the sequence of nonempty subsets of $\{1, \cdots, n\}$ denoted by $\left\{h^{m}\right\}$ is a sequence of points $\left\{x^{r}\right\}$ defined recursively by

$$
\begin{aligned}
& x^{p-1}=x_{i}^{p} \text { if } i \text { h }^{p} \text {, } \\
& f_{i}\left(x_{1}^{p \cdot \mu^{p}}, \cdots, x_{1}^{p+1}, \cdots, x_{\eta}^{p \cdot k^{2}}\right)=z_{1} \text { if } i \in h^{p} \text {, }
\end{aligned}
$$

where for each $i=1, \cdots, n$;

$i$ occurs infinitely often in the sequence $\left\{h^{p}\right\}, k_{i}^{p}$ is a nonnegative integer, $p=0,1, \cdots$, the function $d_{i}(p)=p-k_{i}^{p}$ is isotone, and $\lim _{p \rightarrow \infty} d_{1}(p)=+\infty$.

For an analysis and examples of asynchronous iterations, reference is made to Baudet [1]. The following theorem states a sufficient condition for the monotorte convergence of certain asynchronous iterations.

THEOREM 3.1. Suppose that the conditions of Lemma 2.1 hold. Then the asynchronous iterations $\left\{x^{p}\right\}$ and $\left\{y^{p}\right\}$ corresponding to the same sequences $\left\{h^{p}\right\},\left\{k^{\prime \prime}\right\}$ and starting from $x^{0}$ and $y^{0}$, respectively, are uniquely defined and satisfy

$$
\begin{aligned}
& x^{0} \leqq x^{p} \leq x^{p+1} \leq y^{p+1} \leq y^{p} \leqq y^{0}, \quad p=0,1, \cdots, \\
& \lim _{p \rightarrow x} x^{p}=x^{*} \leqq y^{*}=\lim _{p \rightarrow \infty} y^{p}, \quad F x^{*}=F y^{*}=z .
\end{aligned}
$$

Proof. We recall that by Lemma 2.2 the fixed-point mapping $G$, which is defined by (2.4), is continuous and isotone on $D^{\prime}$. Theorem 3.1 then follows from a convergence result of Miellou mentioned in the Introduction (see [7, Prop. 1]).

LEMMA 3.2. Suppose that the conditions of Lemma 2.1 hold. Then, Fx $x^{\prime \prime} z \leqq F y^{p}$, $p=0,1, \cdots$.

Proof. Since $F$ is oft-diagonally antitone and strictly diagonally isolone, it follows from Theorem 3.1 that for $p=0,1, \cdots$, and $i \in h(p)$

$$
z_{i}=f_{i}\left(y_{1}^{p-k_{1}^{\prime}}, \cdots, y_{i}^{p+1}, \cdots, y_{n}^{p} k_{i n}^{p}\right) \subseteq f_{i}\left(y_{1}^{p}, \cdots, y_{t}^{p+1}, \cdots, y_{n}^{p}\right) \leq f_{1}\left(y^{p}\right) .
$$

For $p=0,1, \cdots$, and $i \notin h(p)$, it follows also that

$$
z_{i} \leq f_{i}\left(y^{0}\right) \leq f_{i}\left(y_{1}^{p}, \cdots, y_{i}^{p+1}, \cdots, y_{n}^{p}\right)=f\left(y^{n}\right),
$$

if $y_{r}^{p+1}$, and

$$
z_{i} \leq f_{i}\left(y_{i}^{p}, \cdots, y_{i}^{p+1}, \cdots, y_{n}^{p}\right\}=f_{i}\left(y^{p}\right),
$$

if $y_{1}^{p+1}=y_{1}^{q+1}, q+1 \leqslant p, \quad z_{1}=f_{i}\left(y_{1}^{q \cdot k_{1}^{1}}, \cdots, y_{1}^{q+1}, \cdots, y_{n}^{q-k_{1}^{k}}\right)$.

Analogousiy, we can show that $F x^{p} \leqq z, p=0,1, \cdots$.

We now consider a particular class of off-diagonally antitone and strictly diagonally isotone mappings: $M$-functions. The rcader is referred to Rheinboldt $[10, \S 2],[1]$, \$2] for a complete study of $M$-functions. Theorem 3.1 and Lemma 3.2 apply in particular to continuous $M$-functions. We now state a global convergence result for continuous surjective $M$-functions. 
TIFORYM 3.3. Let $F: R^{\prime \prime} \rightarrow R^{n}$ be a continuous, surjective $M$-junction. Then, for any $z \in R^{\prime \prime}$, any asynchronows iteration $\left\{x^{n}\right\}$ comverges to the unique solution $x^{*}$ of $F x=z$ for any staring point $x^{\prime \prime} \in R^{\prime \prime}$.

Proof. For given $x^{i 1}, z \in R^{n}$ define the vectors $z, \bar{z}, \underline{x}^{0}, \bar{x}^{(1)} \in R^{n}$ by

$$
\begin{aligned}
& z_{i}=\min \left(f_{i}\left(x^{0}\right), z_{s}\right), \quad \bar{z}_{r}-\max \left(f\left(x^{1)}\right), z_{1}\right), \quad i=\mathrm{t}, \cdots, n, \\
& x^{0}=F^{1}{ }^{2}, \quad \bar{x}^{(0)}=F^{-1}{ }_{z} .
\end{aligned}
$$

Let $\left\{x^{p}\right\},\left\{x^{p}\right\}$, and $\left\{x^{p}\right\}$ denote the asynchronous ifcrations relative to the same probiem and to the same sequences $\left\{h^{p}\right\},\left\{k^{n}\right\}$, and that start from $x^{0}, x^{0}$, and $x^{0}$, respectively. Since a continuous surjective $M$-function is surjectively diagonally isotonc (see $\left[10\right.$, Def. 2.7, Thm. 2.10]), it follows that for $p=0,1, \cdots$, and $i \in h^{r}$ the solutions $x_{i}^{p+1}, \bar{x}_{i}^{p \cdot 1}$, and $x_{i}^{p+1}$ of the equations

$$
\begin{aligned}
& f\left(x_{p}^{p} k_{1}^{p}, \cdots, \underline{x}_{p}^{p+1}, \cdots, x_{n}^{p-k_{p}^{p}}\right)=\bar{z}_{1+} \\
& f_{i}\left(x_{1}^{p} \alpha_{1}, \cdots, \bar{x}_{1}^{p+1}, \cdots, \bar{x}_{n}^{p+k_{1}^{\prime}}\right)=z_{i} \text {, } \\
& f_{i}\left(x_{1}^{p}{ }^{p}, \cdots, x_{i}^{p+1}, \cdots, x_{n}^{p-h !}\right)-z_{i},
\end{aligned}
$$

exist and are unique. It follows also that the asynchronous ilerations $\left\{x^{p}\right\},\left\{x^{p}\right\}$, and $\left\{x^{p}\right\}$ are well defined.

First, we show by induction that

$$
\underline{x}^{\mu} \leqslant x^{p} \div \bar{x}^{p}, \quad p=0,1, \cdots .
$$

From (3.1), $F \underline{x}^{(6} \leq F x^{0} \leq F x^{0}$. Since $F$ is inverse isotone, we have (sec [10, Def. 2.2]) $x^{0} \leq x^{0} \leq x^{0}$. From (3.1) and the inverse isotonictity of $F$ it follows also that $F x^{n} \leq z \leq$ $F x^{0}, x^{0} \leq x^{*} \leq x^{0}$.

Suppose that for some $p \geq 0$

$$
x^{k} \leq x^{k}=\bar{x}^{k} \text { for } 0 \leq k \leq p
$$

Then, if $i t^{n} h^{n}$, it is straightforward that

$$
\underline{x}_{1}^{p+1}=x_{1}^{p}, \quad \bar{x}_{i}^{p+1}=\bar{x}_{1}^{p}, \quad x_{1}^{p+1}=x_{1}^{p}, \quad x_{1}^{p+1} \leq x_{1}^{p+1} \leq \bar{x}_{1}^{p+1} .
$$

If $i \in h^{p}$ from (3.3) and the off-diagonal antitonicity of $F$ it follows that

$$
\begin{aligned}
& f_{i}\left(x_{1}^{p-k_{1}^{\prime}}, \cdots, x_{1}^{p \prime 1}, \cdots, x_{n}^{p} k_{n}^{n}\right)=z_{n}=f_{1}\left(x_{1}^{p} k_{1}^{p}, \cdots, x_{1}^{p+1}, \cdots, x_{n}^{p-k_{n}^{\prime}}\right) \\
& f_{1}\left(\underline{x}_{1}^{p+N_{1}}, \cdots, x_{1}^{p+1}, \cdots, x_{n}^{p} k_{n}^{k}\right) \text {. }
\end{aligned}
$$

Then, by the strict diagonal isotonicity of $F, x_{i}^{p+1} \leq x_{i}^{p+1}$. Analogously, we can show that $x^{m+1} \leq x^{p+1}$. This completes the induction. By Theorem 3.1 we have

$$
\lim _{p \rightarrow x} x^{\prime \prime}=\lim _{p=x} x^{p} \ldots x^{*} \ldots F{ }^{1} z
$$

Then, from (3.2) and (3.4), it follows that $\lim _{p \rightarrow x} x^{p}=x^{*}$.

The results of Theorems 3.1 and 3.3 can be exlended without difficultics to underrelaxed asynchronous iterations.

\section{REFERENCES}

[1] G. M. BAuDt:T, Asynchromous Heratite meshods for mulhprocessors, J. Assoc. Comput. Mach. 25 (1978), pp. 2261-244.

(2) D. P. BERTSt:kns, Distributed dynamic programming, IEEE Trans. Aulomat. Control, 27 (1982), Pp. 610.616. 
[3] D. P. BERTSEKAS ANID D. EL BAZ, Distributed asymchronous relaxation methods jor convex network flow problemts, SIAM J. Control Optim., 25 (1987), pp. 74-85.

[4] D. CHAZAN AND W. MIRANKER, Chaotic relaxation, Lirsar Algebra Appl., 2 (1969), pp. $199-222$.

[5] 3. D. P. DONNELI.Y, Periodic chaotic relaxafion, Linear Algebra Appl., 4 (1971), pp. 117-128.

[6] J. C. MIELLOW, Algorihmes de relaxation chatotique de retards, RAJRO, R-t (1975), pp. $55-82$

[7] _- Jiérations chaotiques à retards; étude de la convergence dans le cas d'espaces partiallement ordonnés, C.R. Acad. Sci. Paris Sér. I Math., 280 (1975), pp. 233-236.

[8] _ Asynchronous iterations and order intervals, in Paratlel Algoritlims and Architectures, NorthHolland, Amsterdam, New York, 1986.

[9] J. M. ORTEGA A YD W. C. RHIEINBOLnT, Iteratite Solution of Nominear Equations in Several Variables, Academic Press, New York, 1970

[10] W. C. RHEINisoLot, On M-functions and their application to nontinear Gauss-Seidel iteratians and io network flows, J. Math. Anal. Appl., 32 (1970), pp. $274-307$.

[11] _. On ciasses of n-dintensionat nontinear mappings generalizing seberal iypes of matrices, in Numerical Solution of Partial Differential Equations-J], B. Hubbard, ed., Academic Press, New York, 1971 , pp. 501-546.

[12] U. SCHENDEL, Introduction fo Numerical Methods for Parallel Conputers, Ellis-Horwood, Chichester. U.K., 1984.

[13] F. StonoDA, Nonlinear inerative methots and parallet computation, Apl. Mat., 21 (1976), pp. 2\$2-262. 
